\title{
Overview of Anti-Seismic Researches of Underground Structures
}

\author{
Ran LIAO \\ Geotechnical engineering, Xipu Campus of Southwest Jiaotong University, Chengdu, Sichuan611756, China
}

\begin{abstract}
With the vigorous development of urban rail transit system, especially the construction of subway system, the safety of subway system draws more and more attention. The study of anti-seismic for underground structures has also become an important problem to be solved in the construction of Metro system. Based on the typical underground structure seismic damage phenomenon, this paper summarizes the seismic characteristics, research methods and design methods of underground structures to offer a guide for engineers.
\end{abstract}

\section{Introduction}

Underground structures have been considered to be of good seismic performance. Seismic design has not been taken into consideration in the design and construction of most of the Metro structures that have been built. Before the Great Hanshin earthquake in Japan in 1995, there were few examples of large underground structures suffered serious damage in the earthquake. People generally had the idea that the damage caused by the earthquake on the underground structure of the building was smaller than that on the over-ground structure. However, during the Great Hanshin earthquake in Kobe City, many underground structures were subject to different degrees of damage, particularly some subway stations and tunnels [1][2][3]. The fact that Kobe city subway stations and tunnelswere severely damaged had brought the huge impact to the traditional concept. This had aroused the attention of scholars and research institutions from all over the world, especially in Japan, to such issues.

In recent years, several strong earthquakes in China,such as the Wenchuan earthquake and the Yushu earthquake, have caused great damages to underground structures [4], which offered more research resources on the behavior of underground structures. With the enlargement of the scale of underground structure construction, the seismic design and safety evaluation of underground structure have been paid more and more attention.

In this paper, typical damages of underground structures are firstly presented, followed by the analysis of the difference between the seismic response of underground and that of over-ground structures. Based on which, the development in research methods and design methods are introduced in the following chapters.

\section{Typical earthquake damages of}

\section{underground structure}

In history, countless underground structures have been damaged in different degrees during the activities of earthquake. Some typical cases in recent years are introduced in the following paragraphs [1][2][3][4][5][6].

The Tangshan 7.8 earthquake in china in 1971 caused serious damages to both over-ground and underground structures, most of which collapsed or were destroyed seriously. The earthquake also caused varying degrees of destruction to underground tunnel, coal mine tunnel and civil air defense works.

The 7.2 magnitude earthquake in Hanshin in Japan in 1995 also broughtgraveaftermath. The earthquake caused the most severe damage to underground structure in Kobe city, including underground railways, underground parking lots, multipurpose underground tunnels and underground shopping malls, etc. Among them, Daikai station and Kamisawa station were completely destroyed. More than half of the middle columns collapsed. Roof collapses and large cracks appeared on the side walks, causing the large-scale settlement of the National Highway Subgrade above the subway. Tens of meters of cracks piled up in the rest of the subway lines, causing traffic paralysis in southern Japan.

In the 1999 Chi-Chi earthquake in Taiwan, only 8 out of 57 mountain tunnels of central Taiwan area were not ruined, and the remaining 49 were damaged in varying degrees. It showed cracks and spalls of the lining, damages of the entrances and exits, bulging and bending of the reinforcement, and displacement of the lining. The cracking of the floor and the collapse of the tunnel also occurred due to slope failure.

During the Wenchuan 8.0 earthquake in china in 2008, 29 of 51 tunnels detected were damaged, of which 18 tunnels were seriously destroyed. Damages of the tunnel near the epicenter were mostly severe, such as the Shaohuiping tunnel, Longxi tunnel, longdongzi tunnel and Zipingpu tunnel.

During the eastern Japan earthquake in 2011, the

\footnotetext{
*Corresponding author: 359465833@qq.com
} 
earthquake along the Pacific coast in northeastern Japan caused ground liquefaction in vast areas from northeast to Kanto area.

\section{Seismic response characteristics of underground structures}

The seismic behaviors of underground structures are quite different from that of the upper structures. The characters of responses in both structures and their main differences are introduced as follows [7][8].

(1) The deformation of underground structure is restrained by the surrounding soil, and the dynamic responses of the structures are not largely influenced by its free vibration characters, while that of the upper structures are dependent on its free vibration characters, particularly the influence of the low modes.

(2) The presence of an underground structure has very little influence on the vibration of the surrounding base (when the ratio of the size of the underground structure to the seismic wavelength is small). However, the existence of the ground structure causes a great disturbance to the seismic oscillation of the free field.

(3) The vibration form of underground structure is greatly influenced by the direction of seismic wave. The deformation and stress of each point of underground structure can change greatly even when the incident direction of seismic wave changes little. The vibration form of ground structure is affected relatively slight by the direction of seismic wave incidence.

(4) There are obvious phase differences of the underground structure in earthquake. While the phase difference of the ground structure in the vibration is not obvious.

(5) In general, the main strain of the underground structure in vibration is not obviously related to the magnitude of earthquake acceleration, but it is closely related to the strain or deformation of surrounding rock and soil medium under earthquake action. As for the upper structure, seismic acceleration is an important factor affecting the dynamic response of the it.

(6) For both underground structures and over-ground structures, the interactions between them and their foundations have an important influence on their dynamic responses, yet with different extent in various ways.

In general, the seismic behavior of the over-ground structures and underground structures are influenced by different factors. The vibration characteristics of the structure and ground vibration field have important impact on their dynamic response. For the over-ground structure, its structure, shape, mass and stiffness changes, namely the self-vibration characteristics change, has great influence on the structural response, while for underground structures, the main contributing element to the reaction is the kinematic characteristics of the surrounding soil, and changes in structural shape generally have relatively little effect on the reaction.

\section{Research methods of seismic}

\section{resistance of underground structures}

The major means to study the seismic performance of underground structures are prototype observation, model experiment and numerical calculation [7][8].

\subsection{Prototype observation}

The prototype observation method refers to the actual observation of the dynamic response and seismic damage of underground structures under seismic action, thus revealing the seismic response characteristics of underground structures, the seismic performance and the mechanism of earthquake damage. Prototype observation includes earthquake observation, field investigation of earthquake damage and full-scale tests. It plays a very important role in seismic research, for it can obtain not only real earthquake records, but also real dynamic responses to verify the result of the calculation method.

\subsection{Model experiment}

The model test method is usually used to study the response characteristics of underground structures through the shock test. It can be divided into artificial source experiment and shaking table experiment. It is the most direct method to study the structure seismic response and failure mechanism in laboratory, so it is widely used [9]. The shaking table test can be classified into ordinary shaking table model test and dynamic centrifuge model test [10], which can simulate the dynamic behavior and seismic performance of a system composed of various structures and soils.

\subsection{Numerical calculation}

With the development of numerical calculation techniques such as finite difference, finite element, boundary element method and discrete element, particularly dynamic numerical calculation, the dynamic numerical methods have been applied to the study of seismic analysis of underground structures. In this method, the entire site is divided into numerical grids with corresponding boundary conditions, then seismic waves are input and dynamic response analysis is performed, then the deformations, stresses and strains in the soil and underground structures will be obtained.

In summary, there is no single means to fully realize the complete and true interpretation and simulation of the dynamic response of underground structures, the seismic performance analysis is developed based on all these three methods.

\section{Simplified design method for seismic design of underground structures}

In order to satisfy the development of underground engineering, simplified seismic analysis method of underground structures were proposed based on the previous researches [11-21]. 


\subsection{Seismic coefficient method}

In 1899, Japanese scholar Oomori Boyoshi put forward the seismic coefficient method based on the static theory. Time dependent seismic forces were firstly equaled to static seismic load, and then static calculation models were used to analyze the internal force and deformation of the structure under seismic loading.

\subsection{Free field deformation method}

In 1960s, Newmark [11] argued that the seismic performance of underground structures was mainly affected by the deformation of surrounding soil, instead of the inertial force that described in the seismic coefficient method. Wang et al [12] and Hashash et al [13] proposed free field deformation method based on this feature. In this method, the free field deformation of the surrounding soil under seismic was directly applied to the structure as the structural deformation. Then the internal force of the structure was calculated, and the structure was designed.

\subsection{Flexibility coefficient method}

The free field deformation method assumes that the deformation of the structure is consistent with the lateral deformation of the surrounding soil, neglecting the uncoordinated deformation between the structure and the surrounding soil. However, in practical engineering, the stiffness of underground structure and the stiffness of surrounding soil are often different. The flexibility coefficient method, also known as soil-structure interaction coefficient method, was established by Penzien et al [14] based on the seismic observation results.

\subsection{Response displacement method}

In 1970s, Japanese scholars found that the decisive factor in the seismic response of underground structures was the deformation of surrounding rock and soil medium instead of the inertial force. And based on whichthe response displacement method was put forward. In this method, the underground structure was assumed as an elastic foundation beam, and the response of underground structure was obtained according to the method of statics.

\subsection{Reaction acceleration method}

The response displacement method belongs to the load-structure method, taking into account three kinds of loads: seismic earth pressure, shear force of soil-structure contact surface and inertial force. While reaction acceleration method belongs to the soil-structure method, and the integral calculation model of soil and underground structure is needed. In this method, only inertia force is considered, which depends on the mass distribution and acceleration of the system. As the mass distribution of the system is definite, it is only necessary to determine the acceleration distribution. Therefore, compared with the reaction displacement method, this method is simpler to use.

\subsection{Pushover analysis method of underground structure}

In early twenty-first century, the static elastoplastic analysis method for underground structures (Pushover analysis method) was proposed by Liu Jing-bo and Li Bin et al [15][16]. The method is to apply an increasing horizontal load to a structure with a certain height distribution structure until a predetermined target structural displacement to analyze the nonlinear response of the structures. This method is used to assess the deformation or capacity of structures and members.

In summary, all these methods reflect the features of behavior of underground structures under horizontal waves to some extent without considering the influence of vertical seismic action, which is an important point to be improved.

\section{Conclusion}

This paper presents the researches on the seismic performance of the underground structures. The main points are as follows:

1. Typical damages of underground structures under earthquakes like collapse of tunnels show the importance of anti-seismic researches.

2. There is a big difference between the seismic performance of over-ground and that of underground structures. The behaviors of underground structures show close relation with the deformation of surrounding soil.

3. Three research methods to analyze the seismic performance for underground structures are presented in this paper.

4. Simplified design methods are introduced. The future trend will be the consideration of the influence vertical seismic action.

\section{References}

1. Yoshida N, Nakamura S. Damage to Dakai subway station during the 1995 Hyogoken-Numnu earthquake and its investigation. Proceedings of Eleventh World Conference on Earthquake Engineering. 1996, 2151:283-300.

2. Iida H, Hiroto T, Yoshida N, et al. Damage to Dakai subway station. Solis and foundations, special issue on geotechnical aspects of the January 171995 Hyogoken-Nambu earthquake. Japanese Geotechnical Society, 1996:283-300.

3. Huo Hong-bin, Bobet A. Seismic design of cut and cover rectangular tunnels-evaluation of observed behavior of Dakai station during Kobe earthquake. Proceedings of 1st World Forum of Chinese Scholars in Geotechnical Engineering, August 20-22, 2003, Tongji University, Shanghai, 456-466.

4. Lin G., Luo S.p., Ni J., Damages of metro structures due to earthquake and corresponding treatment 
measures. Modern Tunneling Technology. 2009, 46(4):36-41.

5. Chen Guo-xing, Chen Su, Du Xin-li, et al. Review of seismic damage, model test, available design and analysis methods of urban underground structures: retrospect and prospect. Journal of Disaster Prevention and Mitigation Engineering, 2016, 36(1):1-23.

6. Penzien J. Seismically induced racking of tunnel linings. Earthquake Engineering Structural Dynamics, 2000, 29(5): 683 - 691 .

7. Li Bin. Theoretical Analysis of SeismicResponse of Underground Subway Structures and lts Application. PHD Thesis. Tsinghua University. 2005.

8. Sun Chao. Study on seismic capability and analysis method of underground subway structures. PHD Thesis. Institute of Engineering Mechanics, China Earthquake Administration. 2009.

9. Tao L.J., Wang P.L., BianJ.. A shaking table test on the representative subway station structure. Jorunal of Beijing Unversity of Technology. 2006, (9):798-801.

10. Kagawa T, Sato M. Centrifuge simulations of large-scale shaking table tests. Journal of Geotechnical and Geoenvironmental Engineering, 2004, 130(7):663-672.

11. Newmark N M. Problems in wave propagation in soil and rock. Proceedings of the International Symposium on Wave Propagation and Dynamic Properties of Earth Materials, 1968.

12. Wang J N. Seismic design of tunnels: A simple state-of-the-art design approach. New York: Parsons Brinckerhoff Quade and Douglas Inc. 1993.

13. Hashash Y, Hook J J,SchmidtB,et al. Seismic design and analysis of underground structures. Tunneling and Underground Space Technology2001,
16(4): $247-293$.

14. Pitilakis K, Tsinidis G. Performance and seismic design of underground structures in earthquake geotechnical engineering design. Springer International Publishing. 2014:279-340.

15. Fishman K L, Mander J B, Richards R, et al. Laboratory study of seismic free-field response of sand $[\mathrm{J}]$ Soil Dynamics and Earthquake Engineering, 1995, 14: 33-43.

16. Liu J.B., Wang W.H., Zhao D.D., et al. Integral response deformation method in seismic analysis of complex section underground structures. China Civil Engineering Journal. 2014, 47(1):134-142.

17. Liu J.B., Liu X.Q., XueY.L.. study on applicability of a pushover analysis method for seismic analysis and design of underground structures. Engineering Mechanics. 2009, 26(1):49-57.

18. Shukla D K, et al. Earthquake load analysis of tunnels and shafts. Proceeding of the 7th World Conference on Earthquake Engineering. Michigan, USA: Univ. of Michigan Press ,1980: 20-28.

19. Choi J S, Lee J S, Kim J M. Nonlinear earthquake response analysis of 2-D underground structure with soil-structure interaction including separation and sliding at interface. 15th ASCE Engineering Mechanics Conference, June 2-5, 2002, Columbia University, New York, 1-8.

20. AFPS/AFTES. Guidelines on Earthquake Design and Protection of Underground Structures. Working Group of the French Association for Seismic Engineering(AFPS) and French Tunneling Association (AF-TES) Version 1, 2001.

21. Kiyomiya O. Earthquake-resistant design feathers of immersed tunnels in Japan. Tunneling and Underground Space Technology. 1995. 10(4):463-457. 